\title{
The Key Role of pH Value in the Synthesis of Titanate Nanotubes-Loaded Manganese Oxides as a Superior Catalyst for the Selective Catalytic Reduction of $\mathrm{NO}$ with $\mathrm{NH}_{3}$
}

\author{
Xiongbo Chen, Chaoping Cen, Zhixiong Tang, Wenhao Zeng, Dingsheng Chen, \\ Ping Fang, and Zhihang Chen

\begin{abstract}
South China Institute of Environmental Sciences, The Ministry of Environmental Protection of PRC, No. 7 West Street, Yuancun, Guangzhou 510655, China
\end{abstract}

Correspondence should be addressed to Chaoping Cen; cenchaoping@scies.org

Received 30 November 2013; Accepted 13 December 2013

Academic Editor: Fan Dong

Copyright (C) 2013 Xiongbo Chen et al. This is an open access article distributed under the Creative Commons Attribution License, which permits unrestricted use, distribution, and reproduction in any medium, provided the original work is properly cited.

\begin{abstract}
Titanate nanotubes (TNTs) synthesized by hydrothermal method were increasingly used as the catalyst support for the selective catalytic reduction (SCR) of NO with ammonia. This paper reports the critical process of postwashing to prepare satisfactory TNTs for the uses of SCR catalysts. Herein, alkaline TNTs (TNTs-AL), acidic TNTs (TNTs-AC), and neutral TNTs (TNTs-NE) were synthesized by controlling washing $\mathrm{pH}$ value. When these TNTs were utilized as the catalyst supports for manganese oxides (Mn/TNTs-AL, Mn/TNTs-AC, and Mn/TNTs-NE), the key role of $\mathrm{pH}$ value was found. Titanate nanosheets, titanate nanorods and titanate nanotubes were dominated in $\mathrm{Mn} / \mathrm{TNTs}-\mathrm{AL}, \mathrm{Mn}$ /TNTs-AC, and $\mathrm{Mn} / \mathrm{TNTs}-\mathrm{NE}$, respectively. $\mathrm{MnO}_{2}$ crystal was observed when using TNTs-AC or TNTs-NE as the support. By contrast, $\mathrm{Mn}_{3} \mathrm{O}_{4}$ and $\mathrm{NaNO}_{3}$ were observed when using TNTs-AL as the support. Mn/TNTs-NE showed the best SCR activity, in line with the largest surface area, the best dispersion, and the most active redox property of manganese oxides. Mn/TNTs-AL showed negligible SCR activity, resulting from the minimum surface area, the $\mathrm{Mn}_{3} \mathrm{O}_{4}$-dominating crystal structure, and the bad dispersion of manganese oxides.
\end{abstract}

\section{Introduction}

In recent years, particular interest has been given to the catalytic application of nanotubular materials including carbon nanotubes (CNTs) and titanate nanotubes (TNTs) $[1,2]$. The tubular channels of these materials act as confined places for both the growth of active particles and catalytic reaction $[3,4]$. In confined circumstances, the redox property of active particles can be tuned and a unique metal-support interaction may occur [5-7]. Furthermore, the interaction between reactants and products within the confined places is enhanced $[1,3,4]$. All these unique properties make nanotubular materials be promising supports for catalysts.

$\mathrm{TiO}_{2}$, mostly nanoparticles, has been one of the most popular supports for both photocatalysts and thermocatalysts [8]. With respect to DeNOx catalysts, which are catalysts for the selective catalytic reduction (SCR) of $\mathrm{NO}_{x}$ with ammonia, $\mathrm{TiO}_{2}$ is preferable to other commonly used supports, for example, $\mathrm{Al}_{2} \mathrm{O}_{3}$ and $\mathrm{SiO}_{2}$ due to the reversible combination with $\mathrm{SO}_{2}$, which is beneficial to preventing $\mathrm{SO}_{2}$ deactivation [9]. The catalyst system $\mathrm{V}_{2} \mathrm{O}_{5}-\mathrm{WO}_{3} / \mathrm{TiO}_{2}$ or $\mathrm{V}_{2} \mathrm{O}_{5}-$ $\mathrm{MoO}_{3} / \mathrm{TiO}_{2}$ is world widely used for removing $\mathrm{NO}_{x}$ from stationary sources. In the meantime, the existing problems including toxicity of vanadium pentoxide, relatively high operation temperature, and $\mathrm{N}_{2} \mathrm{O}$ formation become huge concerns [10-12]. Therefore nonvanadium SCR catalysts have been a hot area of research. Since the successful synthesis by hydrothermal method, titanate nanotubes were promptly used as supports for DeNOx catalysts [2, 13]. Wang et al. found that ceria confined in TNTs showed a superiority in SCR of NO due to the improved redox potential and special adsorption of $\mathrm{NH}_{3}[14,15]$. Nian et al. synthesized $\mathrm{Cu}$ loaded TNTs with high activity for the SCR of NO and believed that the specific feature and layered structure of TNTs facilitated the $\mathrm{Cu}$ dispersion and the formation of solid solution $\mathrm{Cu}_{x} \mathrm{Ti}_{1-x} \mathrm{O}_{2}$ [16]. Yao et al. investigated the performance 
of manganese supported on titanate nanotubes for the lowtemperature SCR of NO and confirmed the superiority of TNTs to $\mathrm{TiO}_{2}$ nanopowder [17]. These TNTs-supported DeNOx catalysts are good attempts to explore non-vanadium SCR catalysts [18].

The hydrothermal synthesis of titanate nanotubes is typically divided into four steps: pretreatment of reagent, hydrothermal reaction, posttreatment washing, and drying $[13,19]$. It is worth noting that the posttreatment washing process is one of the key steps affecting the structure and the composition of the final products [20]. It was proved by Tsai and Teng and Yang et al. that $\mathrm{Na}^{+} \rightarrow \mathrm{H}^{+}$substitution occurred during the washing process and the structural transformation of titanate nanosheets $\rightarrow$ titanate nanotubes $\rightarrow$ anatase $\mathrm{TiO}_{2}$ could be observed, together with the change of elementary composition [20-22]. So it is reasonable that the posttreatment washing process will greatly affect the final property of the TNTs-supported DeNOx catalysts, including the structure, the chemical state of active species, and the SCR activity.

Herein, three different types of TNTs named alkaline TNTs (TNTs-AL), acidic TNTs (TNTs-AC), and neutral TNTs (TNTs-NE) are synthesized and used as catalyst supports for manganese. These catalysts are characterized systematically by SCR activity test, X-ray diffraction (XRD), transmission electron microscopy (TEM), X-ray photoelectron spectroscopy (XPS), and so on. Afterwards, the variation of physicochemical property and catalytic performance are discussed systemically.

\section{Experimental}

2.1. Support Preparation. Commercial available $\mathrm{P} 25 \mathrm{TiO}_{2}$ (Degussa, Germany) was chosen as precursor for the synthesis of TNTs by hydrothermal method. P25 was mixed with $10 \mathrm{~mol} / \mathrm{L} \mathrm{NaOH}$ solution and then transferred to a Teflon lined autoclave. Then, hydrothermal treatment was conducted at $140^{\circ} \mathrm{C}$ for $24 \mathrm{~h}$. Following the hydrothermal reaction, the filtered precipitate was washed according to the special requirements. TNTs-AL were washed with $0.1 \mathrm{~mol} / \mathrm{L}$ $\mathrm{HCl}$ solution to $\mathrm{pH}$ value near 12 . TNTs-AC were washed with $0.1 \mathrm{~mol} / \mathrm{L} \mathrm{HCl}$ solution to $\mathrm{pH}$ value near 2 . TNTs-NE were washed firstly with $0.1 \mathrm{~mol} / \mathrm{L} \mathrm{HCl}$ solution to $\mathrm{pH}$ value near 2 and secondly with distilled water to $\mathrm{pH}$ value near 7 . After the postwashing process, the mixture was filtrated and then dried in oven at $80^{\circ} \mathrm{C}$ for $12 \mathrm{~h}$.

2.2. Catalyst Preparation. Mn/TNTs catalysts with different TNTs supports were prepared by a wetness impregnation technique. $\mathrm{Mn}\left(\mathrm{NO}_{3}\right)_{2} \cdot 4 \mathrm{H}_{2} \mathrm{O}$ was selected as the precursor for manganese oxides. $5 \mathrm{~mL}$ aqueous solution of $50 \mathrm{wt} . \%$ $\mathrm{Mn}\left(\mathrm{NO}_{3}\right)_{2} \cdot 4 \mathrm{H}_{2} \mathrm{O}$ was diluted with $100 \mathrm{~mL}$ deionized water, in which $4 \mathrm{~g}$ of the prepared TNTs was impregnated. The mixture was dried at $80^{\circ} \mathrm{C}$ for $12 \mathrm{~h}$ and then calcined at $400^{\circ} \mathrm{C}$ in air for $3 \mathrm{~h}$.

2.3. Catalyst Characterization. The specific surface areas of the Mn/TNTs catalysts were measured by using a nitrogen adsorption apparatus (ASAP 2020, USA). The samples were degassed at $200^{\circ} \mathrm{C}$ for $6 \mathrm{~h}$ under vacuum. Total pore size distributions were measured from the $\mathrm{N}_{2}$ desorption isotherm using the cylindrical pore model. X-ray photoelectron spectroscopy with $\mathrm{Al} \mathrm{K} \alpha \mathrm{X}$-ray $(\mathrm{hm}=1486.6 \mathrm{eV})$ radiation operated at $150 \mathrm{~W}$ (XPS: Thermo ESCALAB250, USA) was used to investigate the surface properties of the samples. The shift in the binding energy due to relative surface charging was corrected using the $\mathrm{C} 1 \mathrm{~s}$ level at $284.8 \mathrm{eV}$ as an internal standard.

The crystalline phase of the samples was characterized by using an X-ray diffractometer (Rigaku Co., Japan) with $\mathrm{Cu} \mathrm{K} \alpha$ radiation $(\lambda=0.15418 \mathrm{~nm})$. The scattering angle was $2 \theta=$ $10^{\circ}-80^{\circ}$ with step size of $0.02^{\circ} / \mathrm{s}$. The morphology, structure, and grain size of the samples were examined by TEM (JEM2010, Japan).

Temperature programmed reduction (TPR) and temperature programmed desorption (TPD) experiments were carried out using a TP-5085 supplied by Tianjin Xianquan Industry and Trade Development Co. Ltd, China. $0.05 \mathrm{~g}$ of sample was placed in the middle of quartz tube and held in position with silica wool. Prior to TPR experiments, the sample was heated to $400^{\circ} \mathrm{C}$ with a dwelling time of $1 \mathrm{~h}$ and then cooled to $100^{\circ} \mathrm{C}$. The process was operated with purge of flowing $\mathrm{N}_{2}$ gas at the rate of $28.5 \mathrm{~mL} / \mathrm{min}^{-1}$. The TPR runs were then carried out with the linear heating rate $10^{\circ} \mathrm{C} / \mathrm{min}$ in pure $\mathrm{N}_{2}$ containing $4 \% \mathrm{H}_{2}$ at flow rate of $28.5 \mathrm{~mL} / \mathrm{min}$. With respect to TPD experiments, $0.1 \mathrm{~g}$ of sample was pre-treated at $400^{\circ} \mathrm{C}$ for $1 \mathrm{~h}$, cooled to room temperature like TPR, and then saturated with anhydrous $\mathrm{NH}_{3}\left(4 \%\right.$ in $\left.\mathrm{N}_{2}\right)$ at flow rate of $10 \mathrm{~mL} / \mathrm{min}$ for about $30 \mathrm{~min}$. Desorption was carried out by heating the sample in $\mathrm{N}_{2}$ from 70 to $800^{\circ} \mathrm{C}$ with heating rate at $10^{\circ} \mathrm{C} / \mathrm{min}$.

2.4. Catalytic Activity Tests. Catalytic experiments were carried out between 150 and $470^{\circ} \mathrm{C}$ using a fixed-bed quartz reactor with an $8 \mathrm{~mm}$ inner diameter under atmospheric pressure. The feed streams were as follows: $700 \mathrm{ppm} \mathrm{NO}$, 700 ppm $\mathrm{NH}_{3}, 4 \% \mathrm{O}_{2}$, and balanced with $\mathrm{N}_{2}$ as the carrier gas with a total flow rate of $1.8 \mathrm{~L} / \mathrm{min}$. After crushing and sieving, $500 \mathrm{mg}$ of catalyst in the range of 40-60 mesh was used in each experiment. $\mathrm{NO}, \mathrm{NO}_{2}, \mathrm{~N}_{2} \mathrm{O}$, and $\mathrm{O}_{2}$ concentrations were monitored by an infrared gas analyzer (Photon II, Madur Electronics, Austria). All measurements were repeated three times.

\section{Results and Discussion}

3.1. Crystal Structure. Figure 1 shows the $\mathrm{XRD}$ patterns of the three catalysts. The authors reported that the $\mathrm{TiO}_{2}$ crystallites were exfoliated into layered crystalline sheets when the raw P25 was treated in $\mathrm{NaOH}$ aqueous [23]. In the meantime, $\mathrm{Na}-\mathrm{O}-\mathrm{Ti}$ bonds were created on the two sides. During the washing process, $\mathrm{Na}^{+} \rightarrow \mathrm{H}^{+}$substitution and imbalance of $\mathrm{H}^{+}$or $\mathrm{Na}^{+}$ion concentration on two different sides of nanosheets occurred, giving rise to excess surface energy and resulting in bending [2]. From the XRD pattern of the $\mathrm{Mn} / \mathrm{TNTs}-\mathrm{AL}$ sample, strong diffraction peaks for $\mathrm{NaNO}_{3}$ 


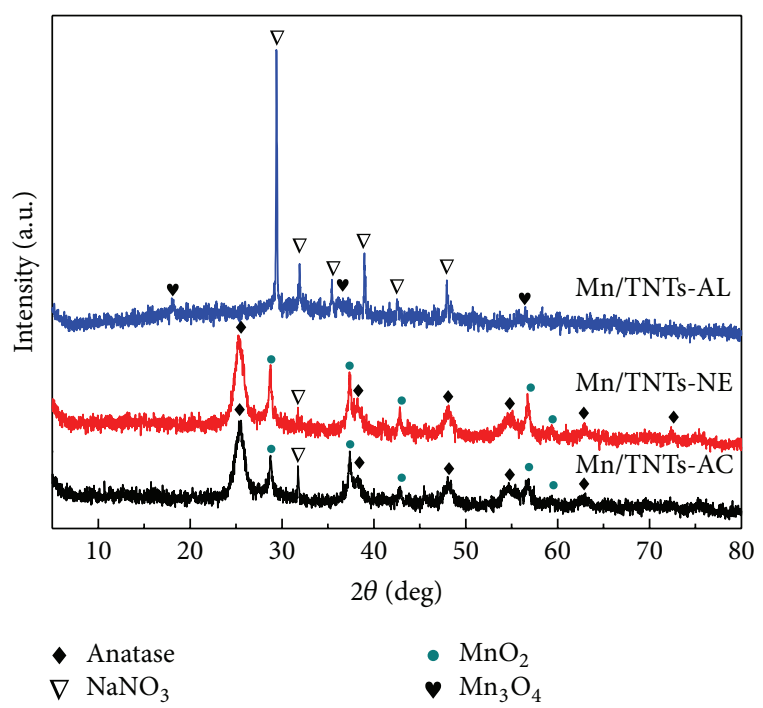

FIGURE 1: XRD patterns of the Mn/TNTs-AL, Mn/TNTs-AC, and $\mathrm{Mn} / \mathrm{TNT}$-NE catalysts.

(PDF\#01-070-1518) were observed. This finding indicates that a large number of $\mathrm{Na}^{+}$still remained in the Mn/TNTs-AL sample. It is because only a part of $\mathrm{Na}^{+}$was substituted by $\mathrm{H}^{+}$if the precipitate was washed with $0.1 \mathrm{~mol} / \mathrm{L} \mathrm{HCl}$ solution to $\mathrm{pH}$ value just near 12 . The residual $\mathrm{Na}^{+}$in titanate structure combined with $\mathrm{NO}_{3}{ }^{-}$groups from manganese nitrate solution. With respect to the manganese oxides, diffraction peaks for $\mathrm{Mn}_{3} \mathrm{O}_{4}$ were observed, whereas those for $\mathrm{MnO}_{2}$ were absent.

Compared with the Mn/TNTs-AL sample, the crystal phase of Mn/TNTs-AC had two obvious changes. Firstly, strong diffraction peaks for anatase (PDF\#01-083-2243) appeared, but most of the diffraction peaks for $\mathrm{NaNO}_{3}$ disappeared (only the peak situated at $31.90^{\circ}$ still remains). These findings indicate that the number of residual $\mathrm{Na}^{+}$ in titanate structure was greatly reduced by washing the precipitates with $\mathrm{HCl}$ solution from $\mathrm{pH}$ value near 12 to $\mathrm{pH}$ value near 2 . In this substitution process, the sodium titanate transformed to anatase [20]. Secondly, diffraction peaks for $\mathrm{Mn}_{3} \mathrm{O}_{4}$ were hardly observed, but diffraction peaks for $\mathrm{MnO}_{2}$ were clearly observed. This phenomenon suggests the different metal-support interaction occurring in Mn/TNTs$\mathrm{AL}$ and Mn/TNTs-AC.

The crystal phase of Mn/TNTs-NE was very similar to that of Mn/TNTs-AC; only small differences on peak intensity could be observed. The diffraction peak situated at $31.90^{\circ}$ for $\mathrm{NaNO}_{3}$ became weaker, but the diffraction peaks for $\mathrm{MnO}_{2}$ got enhanced, which means the residual $\mathrm{Na}^{+}$in the structure of TNTs-AC was further removed by washing with distilled water to $\mathrm{pH}$ value near 7 , and stronger metal-support interaction occurred in the Mn/TNTs-NE sample.

3.2. Morphology and Microstructure. TEM micrographs shown in Figure 2 expressed visual impressions of the morphologies of the catalysts. From Figure 2(a), it can be seen that sodium titanate of the Mn/TNTs-AL sample mainly existed
TABLE 1: Surface area, total pore volume, and mean pore diameter of the Mn/TNTs-AL, Mn/TNTs-AC, and Mn/TNTs-NE samples.

\begin{tabular}{lccc}
\hline Sample & $\begin{array}{c}\text { BET surface area } \\
\left(\mathrm{m}^{2} / \mathrm{g}\right)\end{array}$ & $\begin{array}{c}\text { Pore volume } \\
\left(\mathrm{cm}^{3} / \mathrm{g}\right)\end{array}$ & $\begin{array}{c}\text { Pore size } \\
(\mathrm{nm})\end{array}$ \\
\hline Mn/TNTs-AC & 97.1 & 0.35 & 11.74 \\
Mn/TNTs-NE & 122.4 & 0.53 & 16.45 \\
Mn/TNTs-AL & 8.3 & 0.06 & 19.46 \\
\hline
\end{tabular}

in the form of nanosheets. Nanotubes were hardly observed. This finding indicates that $\mathrm{Na}^{+} \rightarrow \mathrm{H}^{+}$substitution was inadequate, and perhaps the driving force for bending was insufficient. It is reported that almost all the sodium titanate nanosheets will bend to form nanotubes after washing with dilute acid to $\mathrm{pH}$ value reaching 7 [19]. However, most of titanate structure will transform to anatase $\mathrm{TiO}_{2}$ by further washing with dilute acid to $\mathrm{pH}$ value reaching $0-2$, and part of the nanotubular morphology was lost $[24,25]$. In addition, calcination treatment will accelerate the speed of transforming into anatase $\mathrm{TiO}_{2}$ [26]. This is the reason why the assumed nanotubular morphology of the Mn/TNTs-AC sample was absent (Figure 2(b)). Notably, the transformation between titanate nanotube and anatase $\mathrm{TiO}_{2}$ was reversible $[24,25]$. So it is reasonable that the Mn/TNTs-NE sample, which was obtained after washing TNTs-AC with distilled water to $\mathrm{pH}$ value near 7 , impregnating with manganese nitrate and annealing at $400^{\circ} \mathrm{C}$ for $3 \mathrm{~h}$, still maintained nanotube-dominating morphology (Figure 2(c)).

3.3. Physical Characteristics and Chemical Composition. The surface area, total pore volume, and mean pore diameter of the three catalysts are given in Table 1 . The surface areas of Mn/TNTs-AC and Mn/TNTs-NE are large, but that of $\mathrm{Mn} / \mathrm{TNTs}-\mathrm{AL}$ is especially low due to the smooth surface of nanosheets. Large-surface-area feature for titanate materials is advantageous for dispersion of active species [16]. So the dispersion of manganese oxides might be the best in $\mathrm{Mn} / \mathrm{TNT}$-NE and the worst in Mn/TNTs-AL. The pore volume of nanosheets or nanorods is mainly contributed by the interstices between the nanocomposites. The pore volume of nanotubes, on the other hand, is contributed by the internal volume inside the tubes as well as the interstices between the tubes. The Mn/TNTs-NE sample showed the largest pore volume, according well with its best nanotubular morphology among the three catalysts. The pore volume of Mn/TNTsAL was negligibly low, indicating the serious agglomeration of the nanosheets. The pore size, which corresponds to the interstice width, would increase with the size of the constituting nanocomposites. The pore sizes of the three catalysts follow the order Mn/TNTs-AC $<\mathrm{Mn} / \mathrm{TNT}$-NE $<$ $\mathrm{Mn} / \mathrm{TNTs}-\mathrm{AL}$, in line with the size of their constituting nanocomposites (see Figure 2).

As shown in Figure 3(a), typical pore distribution of mesoporous materials could be confirmed in the three catalysts, with most pores concentrating in 2-50 nm. $\mathrm{N}_{2}$ adsorption/desorption isotherms demonstrated in Figure 3(b) gave further information for the pore composition. The hysteretic 


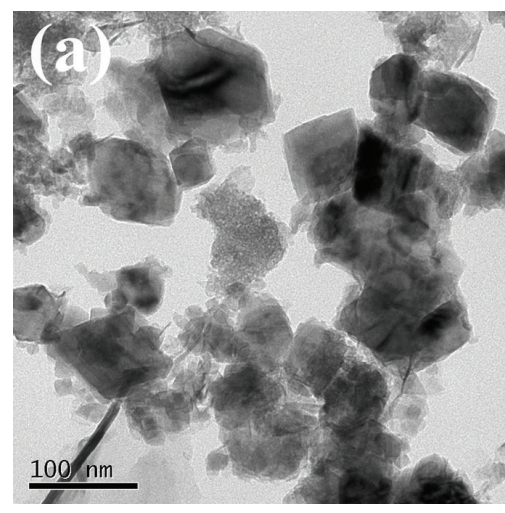

(a)

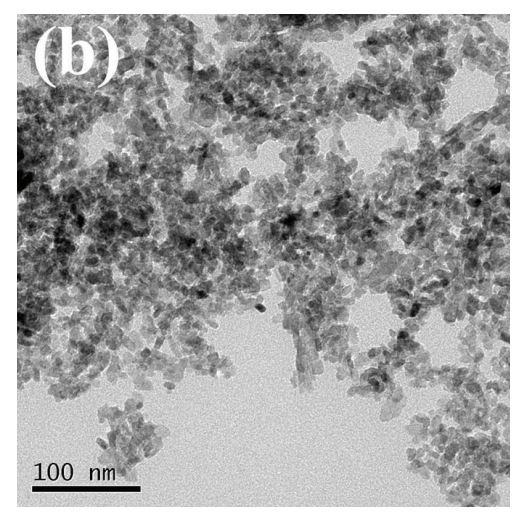

(b)

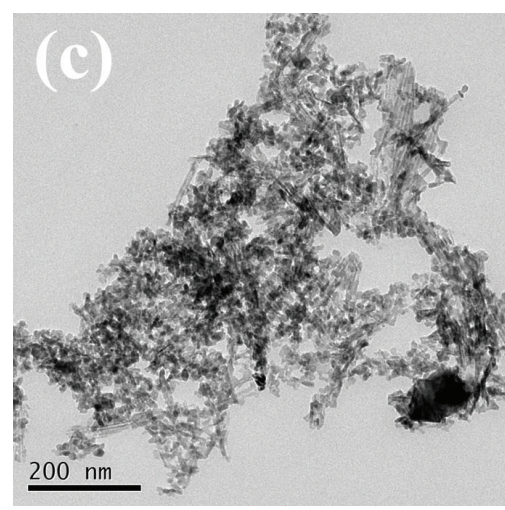

(c)

FIgURE 2: TEM micrographs of (a) Mn/TNTs-AL, (b) Mn/TNTs-AC, and (c) Mn/TNTs-NE.

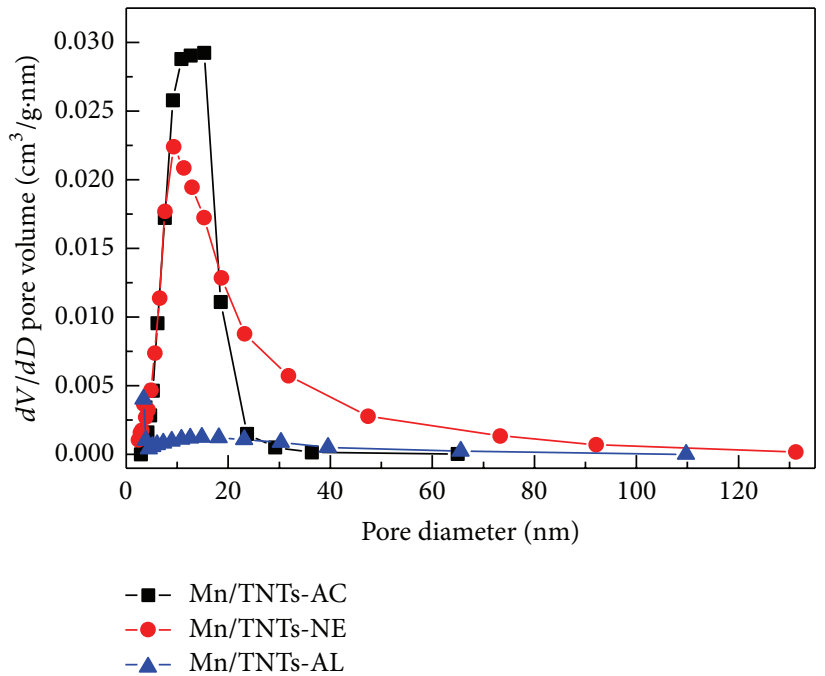

(a)

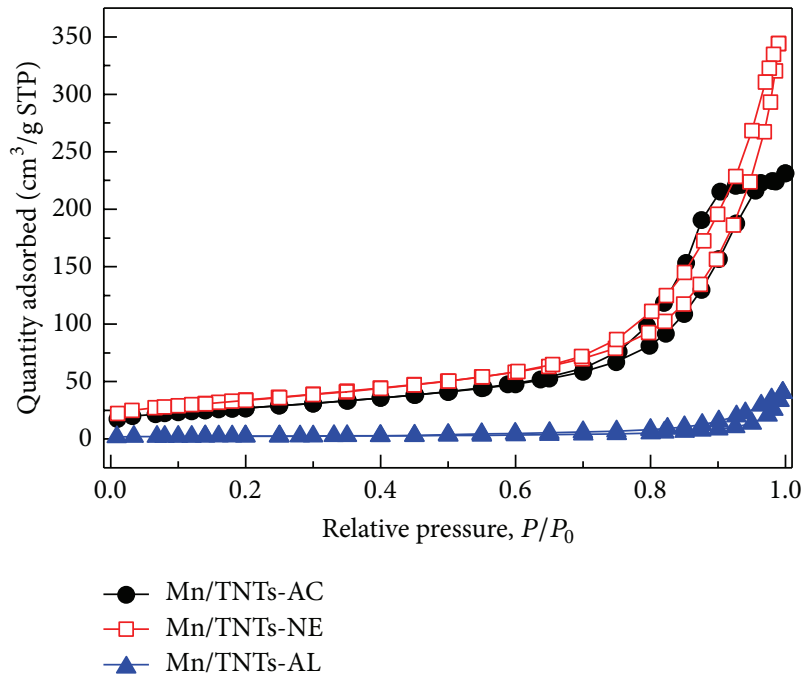

(b)

Figure 3: Pore size distribution (a) and $\mathrm{N}_{2}$ adsorption/desorption isotherms (b) of the three catalysts.

loops of $\mathrm{Mn} / \mathrm{TNTs}-\mathrm{AC}$ could be recognized as type $\mathrm{Hl}$, whereas those of Mn/TNTs-NE and Mn/TNTs-AL could be recognized as type $\mathrm{H} 3$. Type $\mathrm{H} 1$ was always associated with porous materials with uniform shapes and pore size distributions $[27,28]$. By contrast, type $\mathrm{H} 3$ was always associated with porous materials with nonuniform shapes and pore size distributions [28]. In fact, these findings accord well with the TEM results. According to the TEM images, Mn/TNTsAC showed nanorod-dominating morphology. The accumulation of nanorods resulted in uniform mesoporous pores. 


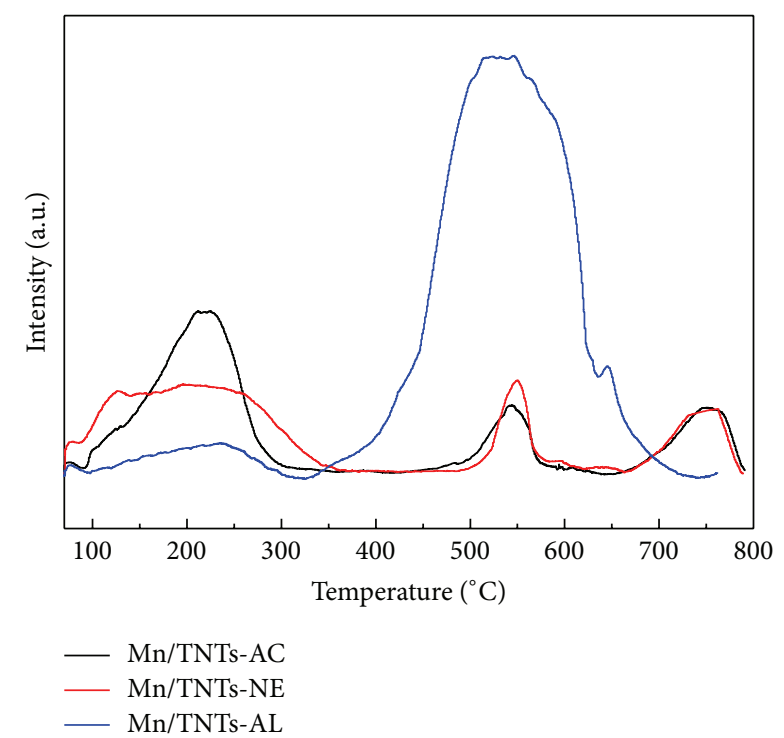

FIgUrE 4: $\mathrm{NH}_{3}$-TPD of the Mn/TNTs-AL, Mn/TNTs-AC, and $\mathrm{Mn} / \mathrm{TNT}$-NE samples.

Mn/TNTs-NE showed nanotube-dominating morphology. The nanotubes were arranged randomly, giving rising to the hollow channels in the tubes and the interstices between the tubes. The inner diameter of TNTs was $c a .2 \mathrm{~nm}$, but the size of the interstices was tens of nanometers [14, 15]. $\mathrm{Mn} / \mathrm{TNTs}-\mathrm{AL}$ showed nanosheet-dominating morphology. These comparatively large nanosheets accumulated to form slits with different sizes.

XPS was utilized to identify the surface nature and atomic concentration of the three catalysts. The atomic concentration of each element was obtained by peak area integral of its narrow spectrum. Previous reports have revealed that the surface atomic concentration of the doped metal obtained with XPS was lower once the metallic species entered the nanotubes $[15,29]$. Since the thickness of the wall of titanate nanotubes was $2-3 \mathrm{~nm}$, which was close to the penetration depth of XPS analysis, manganese located in the tubular channels could only be partially detected. In this paper, the loading amount of manganese for all the three catalysts was designed as the same weight percentage to support, which is equal to $\mathrm{Mn} / \mathrm{Ti}$ molar ratio between 0.28 and 0.39 . However, their atomic concentration shown in Table 2 differed greatly. $\mathrm{Mn} / \mathrm{Ti}$ molar ratio for $\mathrm{Mn} / \mathrm{TNTs}-\mathrm{NE}$ was observed at 0.20 , indicating that lots of manganese in Mn/TNTs-NE entered the tubular channel of titanate nanotubes. By contrast, $\mathrm{Mn}$ /Ti molar ratios for Mn/TNTs-AC and Mn/TNTs-AL were observed at 0.97 and 1.96 , respectively, far exceeding the design value. This is an evidence for the bad dispersion and agglomeration of manganese oxides in Mn/TNTs-AC and $\mathrm{Mn} / \mathrm{TNTs}-\mathrm{AL}$ (especially bad in the latter sample). It is also worth noting that the $\mathrm{Na} / \mathrm{Ti}$ molar ratio of $\mathrm{Mn} / \mathrm{TNTs}-\mathrm{AL}$ reached up to 3.57 , exceeding the other two catalysts by $5-8$ times. This finding that indicates the quantity of residual $\mathrm{Na}^{+}$ in the Mn/TNTs-AL catalyst was much larger than the other two samples. This result accords well with the XRD results revealing the formation of $\mathrm{NaNO}_{3}$ crystal in Mn/TNTs-AL.
TABLE 2: Atomic concentration obtained with XPS.

\begin{tabular}{lcccccc}
\hline Sample & $\mathrm{Ti}(\%)$ & $\mathrm{O}(\%)$ & $\mathrm{Mn}(\%)$ & $\mathrm{Na}(\%)$ & $\mathrm{Mn} / \mathrm{Ti}$ & $\mathrm{Na} / \mathrm{Ti}$ \\
\hline Mn/TNTs-AC & 9.92 & 73.57 & 9.59 & 6.92 & 0.97 & 0.70 \\
Mn/TNTs-NE & 21.36 & 64.14 & 4.24 & 10.26 & 0.20 & 0.48 \\
Mn/TNTs-AL & 5.58 & 63.56 & 10.94 & 19.92 & 1.96 & 3.57 \\
\hline
\end{tabular}

3.4. $\mathrm{NH}_{3}$ Adsorption. As shown in Figure 4, the $\mathrm{NH}_{3}$-TPD profiles of the three catalysts have a common peak situated from $100^{\circ} \mathrm{C}$ to $300^{\circ} \mathrm{C}$. This peak always appeared in the profiles of titanate compounds, belonging to the desorption of ammonia adsorbed at weak acid sites [14]. The intensity of this peak for Mn/TNTs-AL was much weaker than Mn/TNTsAC and Mn/TNTs-NE. The Mn/TNTs-AC and Mn/TNTsNE samples also have another two common peaks centered at $c a .550^{\circ} \mathrm{C}$ and $750^{\circ} \mathrm{C}$, respectively. The former peak could be ascribed to the desorption of chemisorbed ammonia at strong acid sites, and the later peak was generated by the decomposition of residual $\mathrm{NaNO}_{3}$ and Mn-nitrate [30]. As to the $\mathrm{NH}_{3}$-TPD profile of the Mn/TNTs-AL sample, a strong peak ranging within $350-710^{\circ} \mathrm{C}$ was observed. The decomposition of Mn-nitrate and the desorption of chemisorbed ammonia at strong acid sites might contribute to this peak. Considering the large quantity of residuary $\mathrm{NaNO}_{3}$, the continued decomposition of $\mathrm{NaNO}_{3}$ also contributed to this peak greatly.

3.5. Redox Property. The $\mathrm{H}_{2}$-TPR profiles of the three catalysts are shown in Figure 5. According to the previous reports, $\mathrm{MnO}_{2}$ and $\mathrm{Mn}_{2} \mathrm{O}_{3}$ always reduced firstly to $\mathrm{Mn}_{3} \mathrm{O}_{4}$ in the lower temperature range, and secondly to $\mathrm{MnO}$ at higher temperature range [30,31]. With respect to the $\mathrm{H}_{2}$-TPR profiles of the Mn/TNTs-AC and Mn/TNTs-NE catalysts, the two-step reduction was obviously observed. The two reduction peaks for $\mathrm{Mn} / \mathrm{TNTs}-\mathrm{NE}$ centered at $462^{\circ} \mathrm{C}$ and $528^{\circ} \mathrm{C}$ appeared earlier than those for Mn/TNTs-AC centered at $507^{\circ} \mathrm{C}$ and $566^{\circ} \mathrm{C}$. This result suggests that manganese oxides of Mn/TNTs-NE were more active than those of Mn/TNTsAC. Indeed, according to the physical characteristics, a better dispersion of manganese oxides was expected in the surface of Mn/TNTs-NE, leading to the easier reduction at lower temperatures.

Unlike the other two samples, the $\mathrm{H}_{2}$-TPR profile of Mn/TNTs-AL only showed a strong and broad reduction peak. Due to the small surface area of Mn/TNTs-AL, a bad dispersion of manganese oxides trended to occur, giving rise to formation of bulk $\mathrm{MnO}_{x}$. So the reduction peak which appeared in the $\mathrm{H}_{2}$-TPR profile of the Mn/TNTs-AL was in all probability ascribed to the reduction of bulk $\mathrm{MnO}_{x}$.

3.6. SCR Activity. The NO conversion as a function of reaction temperature over the $\mathrm{Mn} / \mathrm{TNTs}-\mathrm{AC}, \mathrm{Mn} / \mathrm{TNTs}-\mathrm{NE}$, and $\mathrm{Mn} / \mathrm{TNTs}-\mathrm{AL}$ catalysts is shown in Figure 6. It can be seen that these three catalysts showed entirely different activity for the SCR of NO. Mn/TNTs-NE showed a good SCR activity even though the activity tests were conducted with a high GHSV at about $100,000 \mathrm{~h}^{-1}$. The NO conversion 


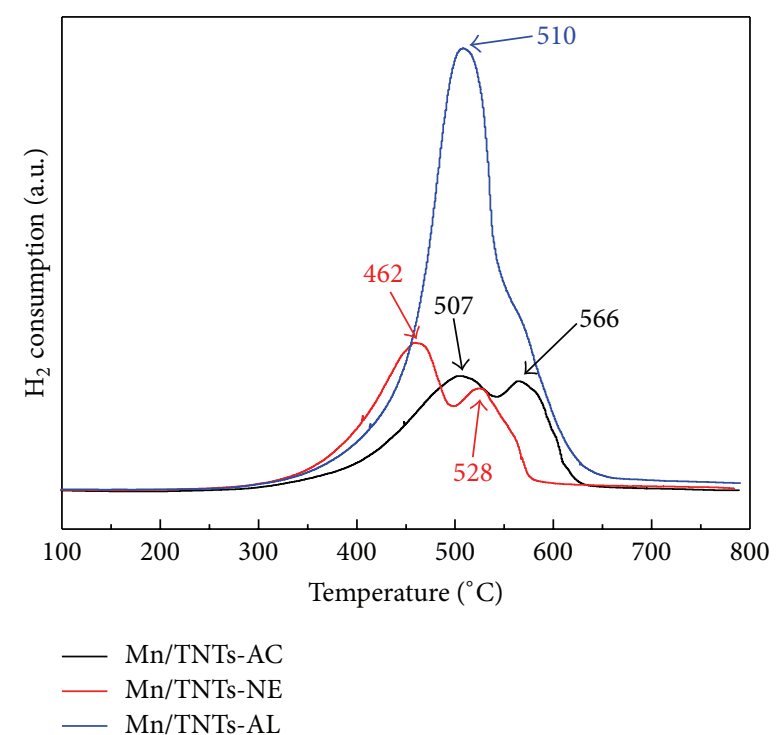

FIgURE 5: $\mathrm{H}_{2}$-TPR of the Mn/TNTs-AL, Mn/TNTs-AC, and $\mathrm{Mn} / \mathrm{TNTs}-\mathrm{NE}$ samples.

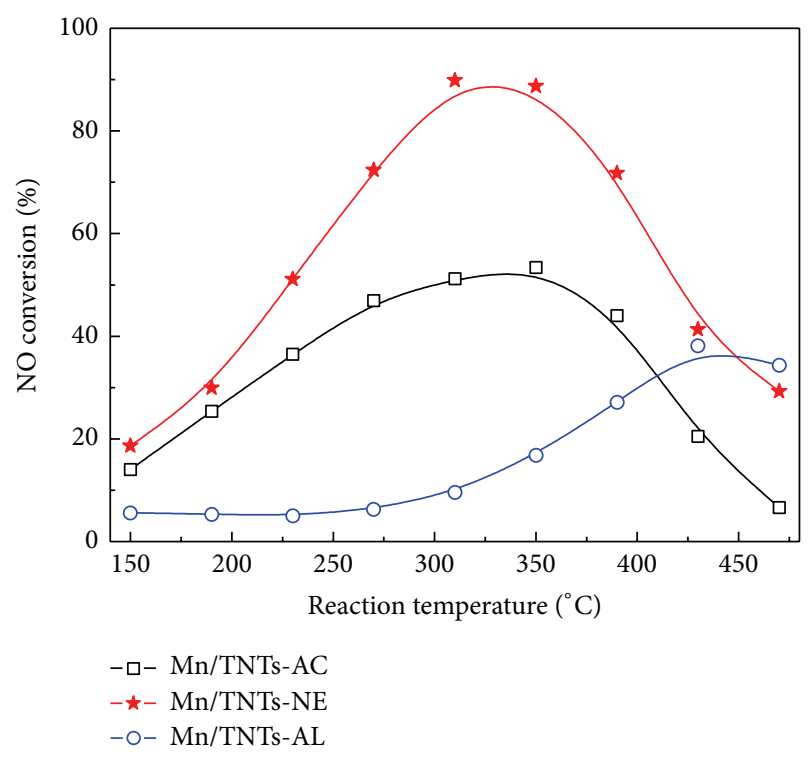

FIGURE 6: Effect of reaction temperature on NO conversion of the $\mathrm{Mn} / \mathrm{TNTs}-\mathrm{AC}, \mathrm{Mn} / \mathrm{TNTs}-\mathrm{NE}$, and Mn/TNTs-AL catalysts. Reaction conditions: $[\mathrm{NO}]=\left[\mathrm{NH}_{3}\right]=600 \mathrm{ppm},\left[\mathrm{O}_{2}\right]=3.5 \%$, balanced $\mathrm{N}_{2}$, catalyst $0.5 \mathrm{~g}$, and GHSV about $100,000 \mathrm{~h}^{-1}$.

for $\mathrm{Mn} / \mathrm{TNTs}-\mathrm{NE}$ at $300^{\circ} \mathrm{C}$ to $350^{\circ} \mathrm{C}$ reached $90 \%$. The SCR activity of Mn/TNTs-AC is worse in comparison. Even worse, the NO conversion of Mn/TNTs-AL was negligibly low in the main reaction temperature range of $200^{\circ} \mathrm{C}$ to $400^{\circ} \mathrm{C}$.

Based on the above discussions, the composition, dispersion, and redox property of manganese oxides might have affected the SCR activity. Two types of crystal phase for manganese oxides were formed due to different metalsupport interaction. $\mathrm{MnO}_{2}$ was observed in Mn/TNTs$\mathrm{AC}$ and $\mathrm{Mn} / \mathrm{TNTs}-\mathrm{NE}$, whereas $\mathrm{Mn}_{3} \mathrm{O}_{4}$ was observed in
$\mathrm{Mn} / \mathrm{TNT}$-AL. It was reported that the SCR activity of $\mathrm{MnO}_{2}$ was better than $\mathrm{Mn}_{3} \mathrm{O}_{4}$ [32]. Mn/TNTs-NE possessed the maximum surface area, resulting in the best dispersion of manganese oxides. In addition, partial manganese oxides of Mn/TNTs-NE entered into the tubular channels; thus the redox property of manganese oxides might be tuned and a unique metal-support interaction might occur [5-7], leading to the enhancement of activity. By contrast, Mn/TNTs-AL showed especially low surface area, thus giving rise to the worse dispersion of manganese oxides.

\section{Conclusion}

Alkaline TNTs, acidic TNTs, and neutral TNTs were synthesized by hydrothermal method with controlled postwashing treatment. Manganese oxides were loaded on these TNTs and used for the SCR of NO with ammonia. TEM images revealed that titanate nanosheets, titanate nanorods, and titanate nanotubes were dominated in Mn/TNTs-AL, Mn/TNTs-AC and $\mathrm{Mn}$ /TNTs-NE, respectively. $\mathrm{MnO}_{2}$ crystal was observed in $\mathrm{Mn}$ /TNTs-AC, and Mn/TNTs-NE. $\mathrm{Mn}_{3} \mathrm{O}_{4}$ and $\mathrm{NaNO}_{3}$, on the other hand, were observed in Mn/TNTs-AL. Among the three catalysts, Mn/TNTs-NE showed the largest surface area, the best dispersion of active species, and the most active redox property of manganese oxides, and thus had the best SCR activity. By contrast, Mn/TNTs-AL showed especially low surface area, $\mathrm{Mn}_{3} \mathrm{O}_{4}$-dominating crystal structure, and bad dispersion of manganese oxides and unsurprisingly showed negligible SCR activity.

\section{Acknowledgments}

This research was financially supported by the National Natural Science Foundation of China (NSFC-51306068), the Key Laboratory for Water and Air Pollution Control, Guangdong Province (no. 2011A060901002), the Central-Level Nonprofit Scientific Institutes for Basic R\&D Operations (PM-zx021201211-106), and the National High Technology Research and Development Program (863) of China (2012AA062505).

\section{References}

[1] X. Pan and X. Bao, "Reactions over catalysts confined in carbon nanotubes," Chemical Communications, no. 47, pp. 6271-6281, 2008.

[2] D. V. Bavykin, J. M. Friedrich, and F. C. Walsh, "Protonated titanates and $\mathrm{TiO}_{2}$ nanostructured materials: synthesis, properties, and applications," Advanced Materials, vol. 18, no. 21, pp. 2807-2824, 2006.

[3] X. Pan, Z. Fan, W. Chen, Y. Ding, H. Luo, and X. Bao, "Enhanced ethanol production inside carbon-nanotube reactors containing catalytic particles," Nature Materials, vol. 6, no. 7, pp. 507511, 2007.

[4] Q. Fu, W.-X. Li, Y. Yao et al., "Interface-confined ferrous centers for catalytic oxidation," Science, vol. 328, no. 5982, pp. 1141-1144, 2010.

[5] W. Chen, X. Pan, M.-G. Willinger, D. S. Su, and X. Bao, "Facile autoreduction of iron oxide/carbon nanotube encapsulates," 
Journal of the American Chemical Society, vol. 128, no. 10, pp. 3136-3137, 2006.

[6] W. Chen, X. Pan, and X. Bao, "Tuning of redox properties of iron and iron oxides via encapsulation within carbon nanotubes," Journal of the American Chemical Society, vol. 129, no. 23, pp. 7421-7426, 2007.

[7] W. Chen, Z. Fan, X. Pan, and X. Bao, "Effect of confinement in carbon nanotubes on the activity of Fischer-Tropsch iron catalyst," Journal of the American Chemical Society, vol. 130, no. 29, pp. 9414-9419, 2008.

[8] H. G. Yang, C. H. Sun, S. Z. Qiao et al., "Anatase $\mathrm{TiO}_{2}$ single crystals with a large percentage of reactive facets," Nature, vol. 453, no. 7195, pp. 638-641, 2008.

[9] G. Busca, L. Lietti, G. Ramis, and F. Berti, "Chemical and mechanistic aspects of the selective catalytic reduction of $\mathrm{NO}_{X}$ by ammonia over oxide catalysts: a review," Applied Catalysis $B$, vol. 18, no. 1-2, pp. 1-36, 1998.

[10] W. Shan, F. Liu, H. He, X. Shi, and C. Zhang, "Novel ceriumtungsten mixed oxide catalyst for the selective catalytic reduction of $\mathrm{NO}_{X}$ with $\mathrm{NH}_{3}$," Chemical Communications, vol. 47, no. 28, pp. 8046-8048, 2011.

[11] F. Liu, H. He, and C. Zhang, "Novel iron titanate catalyst for the selective catalytic reduction of $\mathrm{NO}$ with $\mathrm{NH}_{3}$ in the medium temperature range," Chemical Communications, no. 17, pp. 2043-2045, 2008.

[12] G. Qi and R. T. Yang, "A superior catalyst for low-temperature NO reduction with $\mathrm{NH}_{3}$," Chemical Communications, no. 7, pp. 848-849, 2003.

[13] T. Kasuga, M. Hiramatsu, A. Hoson, T. Sekino, and K. Niihara, "Formation of titanium oxide nanotube," Langmuir, vol. 14, no. 12, pp. 3160-3163, 1998.

[14] H. Wang, X. Chen, X. Weng, Y. Liu, S. Gao, and Z. Wu, "Enhanced catalytic activity for selective catalytic reduction of $\mathrm{NO}$ over titanium nanotube-confined $\mathrm{CeO}_{2}$ catalyst," Catalysis Communications, vol. 12, no. 11, pp. 1042-1045, 2011.

[15] X. Chen, H. Wang, Z. Wu, Y. Liu, and X. Weng, "Novel $\mathrm{H}_{2} \mathrm{Ti}_{12} \mathrm{O}_{25}$-confined $\mathrm{CeO}_{2}$ catalyst with remarkable resistance to alkali poisoning based on the 'shell protection effect,"' The Journal of Physical Chemistry C, vol. 115, no. 35, pp. 17479-17484, 2011.

[16] J.-N. Nian, S.-A. Chen, C.-C. Tsai, and H. Teng, "Structural feature and catalytic performance of $\mathrm{Cu}$ species distributed over $\mathrm{TiO}_{2}$ nanotubes," The Journal of Physical Chemistry B, vol. 110, no. 51, pp. 25817-25824, 2006.

[17] Y. Yao, S.-L. Zhang, Q. Zhong, and X.-X. Liu, "Low temperature selective catalytic reduction of NO over manganese supported on $\mathrm{TiO}_{2}$ nanotubes," Journal of Fuel Chemistry and Technology, vol. 39, no. 9, pp. 694-701, 2011.

[18] P. Forzatti, "Present status and perspectives in de- $\mathrm{NO}_{X}$ SCR catalysis," Applied Catalysis A, vol. 222, no. 1-2, pp. 221-236, 2001.

[19] X. Sun and Y. Li, "Synthesis and characterization of ionexchangeable titanate nanotubes," Chemistry, vol. 9, no. 10, pp. 2229-2238, 2003.

[20] C.-C. Tsai and H. Teng, "Structural features of nanotubes synthesized from $\mathrm{NaOH}$ treatment on $\mathrm{TiO}_{2}$ with different posttreatments," Chemistry of Materials, vol. 18, no. 2, pp. 367-373, 2006.

[21] C.-C. Tsai and H. Teng, "Regulation of the physical characteristics of titania nanotube aggregates synthesized from hydrothermal treatment," Chemistry of Materials, vol. 16, no. 22, pp. 4352-4358, 2004.
[22] J. Yang, Z. Jin, X. Wang et al., "Study on composition, structure and formation process of nanotube $\mathrm{Na}_{2} \mathrm{Ti}_{2} \mathrm{O}_{4}(\mathrm{OH})_{2}$," Dalton Transactions, no. 20, pp. 3898-3901, 2003.

[23] B. D. Yao, Y. F. Chan, X. Y. Zhang, W. F. Zhang, Z. Y. Yang, and N. Wang, "Formation mechanism of $\mathrm{TiO}_{2}$ nanotubes," Applied Physics Letters, vol. 82, no. 2, pp. 281-283, 2003.

[24] H. Y. Zhu, Y. Lan, X. P. Gao et al., "Phase transition between nanostructures of titanate and titanium dioxides via simple wetchemical reactions," Journal of the American Chemical Society, vol. 127, no. 18, pp. 6730-6736, 2005.

[25] H. Zhu, X. Gao, Y. Lan, D. Song, Y. Xi, and J. Zhao, "Hydrogen titanate nanofibers covered with anatase nanocrystals: a delicate structure achieved by the wet chemistry reaction of the titanate nanofibers," Journal of the American Chemical Society, vol. 126, no. 27, pp. 8380-8381, 2004.

[26] D. V. Bavykin, M. Carravetta, A. N. Kulak, and F. C. Walsh, "Application of magic-angle spinning NMR to examine the nature of protons in titanate nanotubes," Chemistry of Materials, vol. 22, no. 8, pp. 2458-2465, 2010.

[27] J. Liu, Y. Liu, Z. Wu, X. Chen, H. Wang, and X. Weng, "Polyethyleneimine functionalized protonated titanate nanotubes as superior carbon dioxide adsorbents," Journal of Colloid and Interface Science, vol. 386, no. 1, pp. 392-397, 2012.

[28] K. S. W. Sing, "Reporting physisorption data for gas/solid systems with special reference to the determination of surfancearea and porosity," Pure and Applied Chemistry, vol. 54, no. 11, pp. 2201-2218, 1982.

[29] X. Chen, S. Cao, X. Weng, H. Wang, and Z. Wu, "Effects of morphology and structure of titanate supports on the performance of ceria in selective catalytic reduction of NO," Catalysis Communications, vol. 26, pp. 178-182, 2012.

[30] M. Pourkhalil, A. Z. Moghaddam, A. Rashidi, J. Towfighi, and Y. Mortazavi, "Preparation of highly active manganese oxides supported on functionalized MWNTs for low temperature $\mathrm{NO}_{X}$ reduction with $\mathrm{NH}_{3}$," Applied Surface Science, vol. 279, pp. 250259, 2013.

[31] K. Zhuang, J. Qiu, F. Tang, B. Xu, and Y. Fan, "The structure and catalytic activity of anatase and rutile titania supported manganese oxide catalysts for selective catalytic reduction of $\mathrm{NO}$ by $\mathrm{NH}_{3}$," Physical Chemistry Chemical Physics, vol. 13, no. 10, pp. 4463-4469, 2011.

[32] L. Wang, B. Huang, Y. Su et al., "Manganese oxides supported on multi-walled carbon nanotubes for selective catalytic reduction of $\mathrm{NO}$ with $\mathrm{NH}_{3}$ : catalytic activity and characterization," Chemical Engineering Journal, vol. 192, pp. 232-241, 2012. 

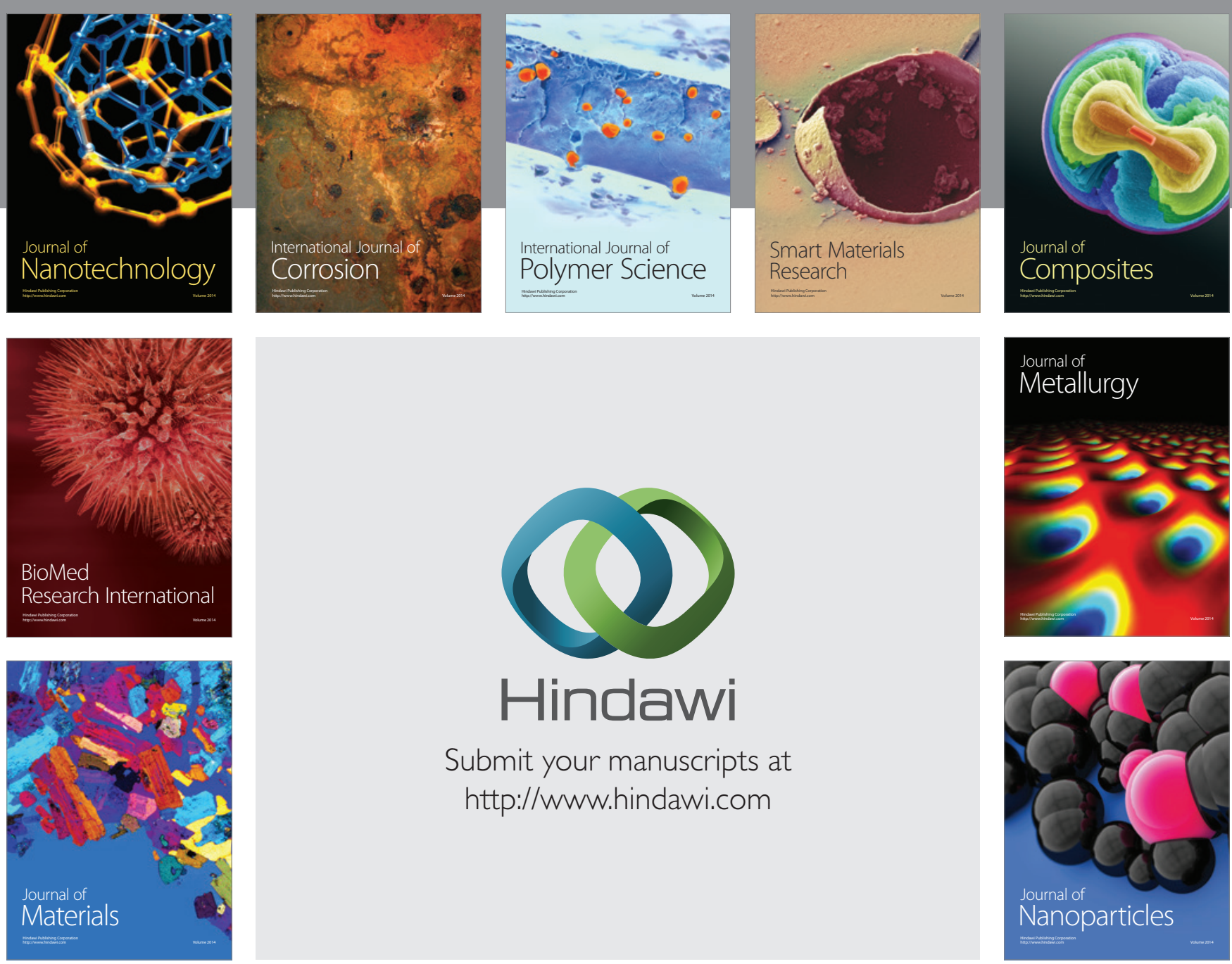

Submit your manuscripts at http://www.hindawi.com
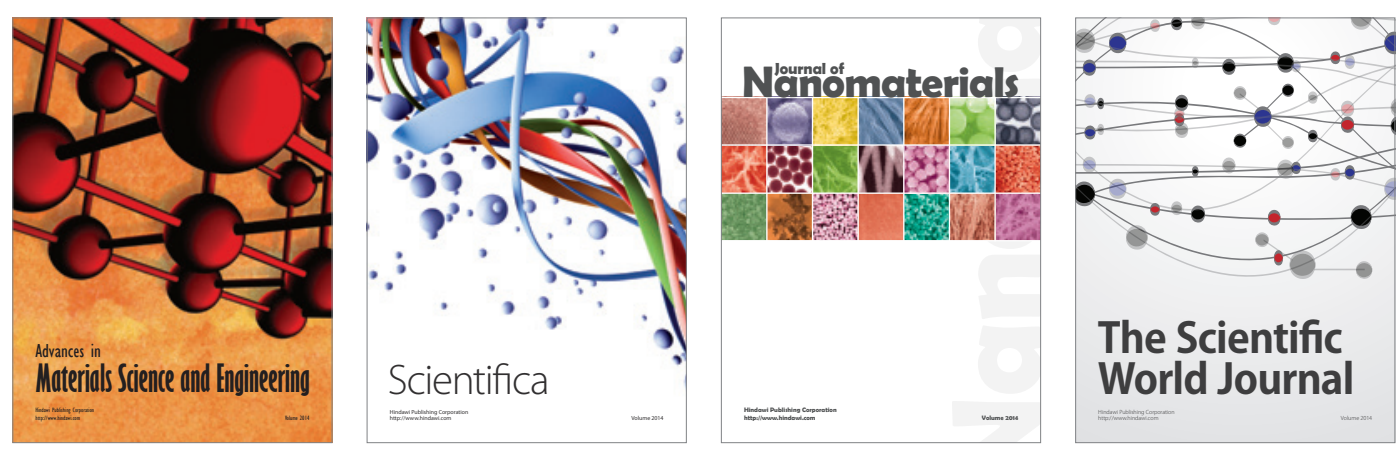

\section{The Scientific World Journal}
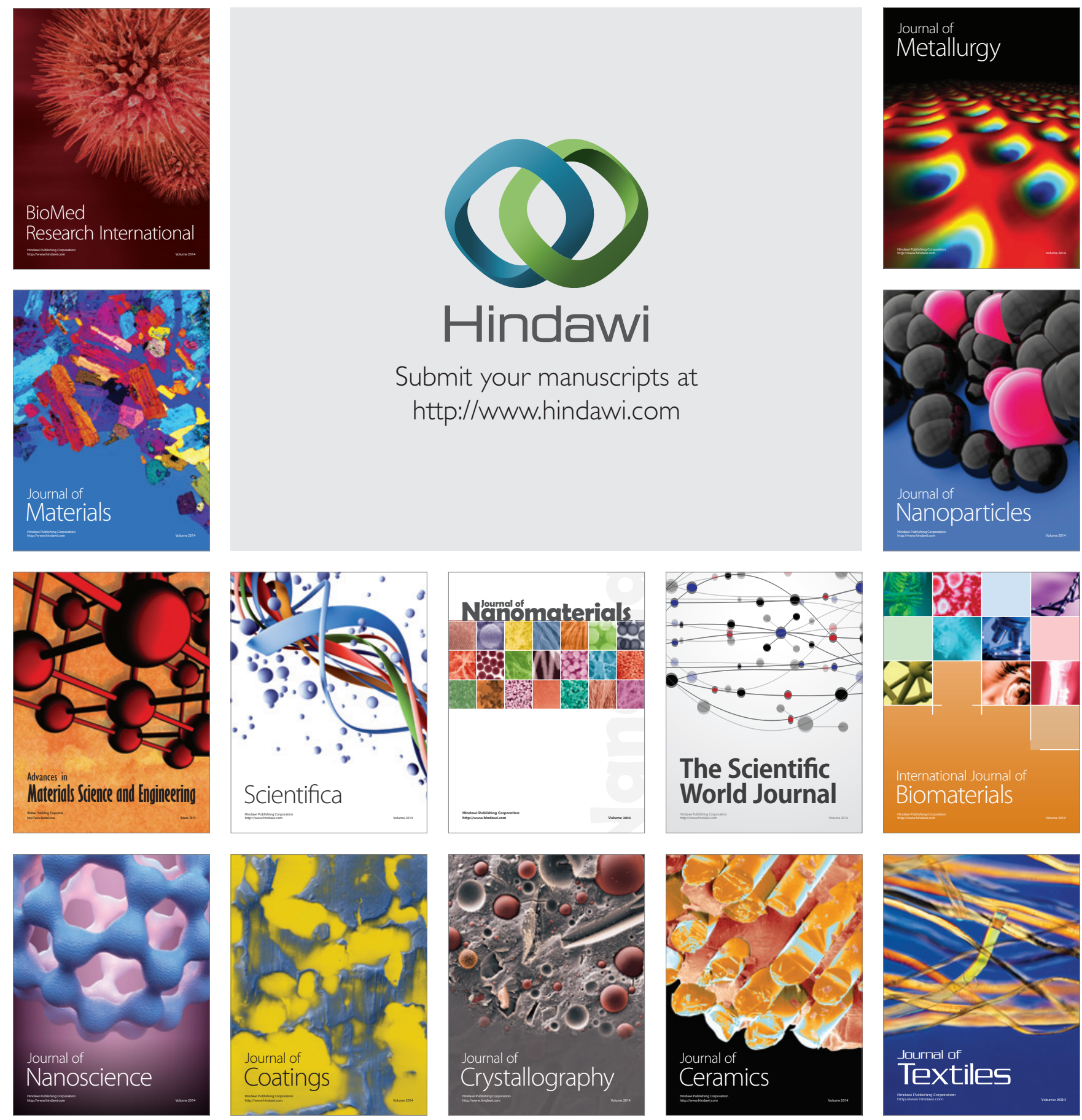